\title{
Determinanten der sozialen Lage: Arbeitsmarkt versus Familie
}

\section{Thomas Klein}

Theresienstraße 128, D-8000 München 2

Zusammenfassung: In dem vorliegenden Beitrag wurde der Frage nachgegangen, inwieweit aus der Stellung auf dem Arbeitsmarkt auch auf die Verteilung von Armut geschlossen werden kann, die früher unter dem Einfluß familialer Faktoren analysiert wurde. Im wesentlichen wurden erstmals auf der Basis einer empirischen Mikrosimulation drei Fragen untersucht. Welchen Einfluß haben Arbeitsmarktsegmentierung versus Familie auf die sog. Erwerbstätigenarmut? Wie wirken sich beide Faktoren auf das Risiko von Verarmung und Abstieg im Fall von Arbeitslosigkeit aus? Inwieweit kumulieren Arbeitslosigkeits- und Verarmungsrisiken im Hinblick auf Merkmale der Arbeitsmarktstellung und des Familienzusammenhangs?

Im Ergebnis läßt sich festhalten, daß die Erwerbstätigenarmut und das Verarmungsrisiko bei Arbeitslosigkeit - trotz deutlicher Segmentierungseinflüsse - stark auch von dem familialen Kontext bestimmt werden. Hinsichtlich der Verknüpfung von Arbeitslosigkeits- und Verarmungsrisiko im Fall von Arbeitslosigkeit kann man sogar teilweise von kompensierenden Einflüssen ausgehen.

\section{Einleitung}

Soziale Ungleichheit und Armut werden häufig auf der Grundlage von Arbeitsmarkttheorien diskutiert und aus dem Geschehen auf dem Arbeitsmarkt abgeleitet. Wiederholt ist dabei in den letzten Jahren auch in dieser Zeitschrift der Versuch unternommen worden, das Verarmungsrisiko mit der Segmentierung des Arbeitsmarktes in Verbindung zu bringen (Heinze et al. 1981; 1983; Buttler et al. 1978). Damit sollte die ,Neue Soziale Frage', wie seit Geißler (1976) ein erhöhtes Verarmungsrisiko der Nichtproduzenten und Nichtorganisierten genannt wird, auf ihren alten Kern, die Armut der Arbeiterschaft als die (alte) soziale Frage des 19. Jahrhunderts, zurückgeführt werden. Eine solche Rückführbarkeit von Verarmungsprozessen auf das Arbeitsmarktgeschehen wäre von großer praktischer Bedeutung für die Sozialpolitik: Zur Bekämpfung der Armut müßte in die Allokationsregeln des Arbeitsmarktes eingegriffen werden, und es müßte eine stärkere Entkoppelung von Arbeitsmarktstatus und sozialstaatlicher Absicherung angestrebt werden (Heinze et al. 1981).

Zahlreiche Untersuchungen belegen, daß beispielsweise die in der Bundesrepublik Deutschland nunmehr schon seit einigen Jahren anhaltend hohe Massenarbeitslosigkeit zu recht für umfangreiche Verarmungs- und Abstiegsprozesse verantwortlich gemacht wird (Adamy/Steffen 1984; Albrecht et al. 1977; Bäcker 1983; Balsen et al. 1984; Breuer et al. 1984; Brinkmann 1976; 1984; Brinkmann/Schober 1982; Brinkmann/Spitznagel 1984; Büchtemann 1982; 1985; Büchtemann/von Rosenbladt 1981; 1983; Buttler et al. 1978; Cramer 1980; CramerSchäfer 1981; Furmaniak 1984; Gölter o. J.; Hau- ser et al. 1985; Klein 1985; 1987; Kortmann 1977; Muhr 1984; Naegele 1979; Rosenbladt, von/Büchtemann 1980; Schäfer 1983; Scholz 1983; Wacker 1977; Welzmüller 1982). Trotzdem stellt sich die Frage, ob gerade diejenigen, die auf dem Arbeitsmarkt und aufgrund von Arbeitsmarktstrukturierungen benachteiligt sind, in besonderem Maße auch von Armut bedroht sind. Kohl und Leisering (1982: 420) sprechen davon, daß die Verteilungsmechanismen des Arbeitsmarktes zum Teil durch die familiale Einbindung des einzelnen, durch das „Verteilungssystem Familie“, aufgehoben würden. Die Lebenslage des einzelnen resultiere aus dem Zusammenwirken mehrerer Verteilungssysteme, wobei auch die innerfamiliären Umverteilungen von großer Bedeutung sind.

Zielsetzung dieses Beitrags ist eine empirische Beleuchtung der Kontroverse zwischen Heinze et al. (1981; 1983) und Kohl und Leisering (1982) im Hinblick auf die Frage nach der relativen Bedeutung der Verteilungssysteme Arbeitsmarkt und Familie auf die Lebenslage des Individuums. Im Mittelpunkt stehen dabei die Einflüsse, welche Arbeitsmarkt- und Familienstrukturen auf die Lebenslage bei Erwerbstätigkeit und auf das Risiko der Verarmung und des sozialen Abstiegs durch Arbeitslosigkeit ausüben.

\section{Arbeitsmarktsegmentierung als Ursache von Verarmung bei Arbeitslosigkeit - Thesen und Gegenthesen}

Ein enger Zusammenhang zwischen Arbeitsmarktlage und Verarmungsrisiko wird mit der Theorie des segmentierten Arbeitsmarktes (z. B. Sengen- 
berger 1978) begründet. Hiernach spaltet sich der Arbeismarkt in einen primären bzw. ,internen“" Arbeitsmarkt mit hohen Löhnen, guten Arbeitsbedingungen, Beschäftigungssicherheit und der Möglichkeit innerbetrieblicher Weiterqualifikation und einen sekundären bzw. „externen“ Arbeitsmarkt mit niedrigen Löhnen, schlechten Arbeitsbedingungen, instabilen Beschäftigungen und geringen beruflichen Mobilitätschancen. Für diese Segmentierung sind betriebliche Rationalitätsprinzipien und unterschiedliche Qualifikationsanforderungen, verbunden mit einer unterschiedlich hohen Austauschbarkeit der Arbeitskräfte, verantwortlich; diese Mechanismen sollen hier nicht weiter diskutiert werden. $\mathrm{Zu}$ dem benachteiligten sekundären Arbeitsmarktsegment werden in der Bundesrepublik vor allem Frauen, Unqualifizierte, Jugendliche und Ältere gerechnet. Auch ohne theoretische Erklärung können diese Gruppen jedenfalls als Problemgruppen des Arbeitsmarktes bezeichnet werden.

Heinze et al. (1981) sehen „vorrangig“ die am Arbeitsmarkt benachteiligten Arbeitnehmer des zweiten oder unteren Arbeitsmarktsegments Frauen, Niedrigqualifizierte, Jugendliche und Ältere - einer gleichzeitig erhöhten Verarmungsgefahr ausgesetzt:

- Einerseits stellten die niedrigen Verdienstchancen der Erwerbstätigen im sekundären Arbeitsmarktsegment selbst eine Existenzgefährdung dar, die sich in Form der Erwerbstätigenarmut, der sog. „working poor“, manifestiert (Heinze et al. 1983: 165).

- Soweit ehemalige Erwerbstätige aus dem Erwerbsleben ausgeschieden sind, bewirke andererseits die Koppelung vieler Transfereinkommen wie Rente oder Arbeitslosenunterstützung an das frühere Arbeitseinkommen ein erhöhtes Verarmungsrisiko für die ehemals im sekundären Arbeitsmarkt mit niedrigem Lohn Beschäftigten. Die Leistungen des Systems der sozialen Sicherung bei Nichterwerbstätigkeit bemessen sich häufig nach zuvor erbrachten Beiträgen, wodurch das Sozialeinkommen als eine aus dem Arbeitseinkommen abgeleitete Einkommensquelle erscheint, in der sich die Arbeitsmarktstrukturierung fortsetzt (Heinze et al. 1983: 161).

- Schließlich mache sich die von der Segmentzugehörigkeit abhängige Marktlage vor allem in konjunkturell schwachen Situationen bemerkbar, in denen die Betriebe zunächst die austauschbaren Randbelegschaften abbauen, deren Wiederbeschäftigungschance sich gleichzeitig drastisch ver- schlechtert. Der im sekundären Arbeitsmarktsegment ohnehin niedrigere Verdienst sei obendrein sehr viel unsicherer. In Zeiten hoher Arbeitslosigkeit trage diese Unsicherheit zusätzlich zu einer erhöhten Armut bei. Beide Nachteile - die unsichere Arbeitsmarktstellung, verbunden mit einem höheren Arbeitslosigkeitsrisiko, und die schlechtere Absicherung im Fall von Arbeitslosigkeit - kumulierten daher zu einem höheren Verarmungsrisiko der Arbeitnehmer des zweiten Arbeitsmarktsegments.

Trotz aller Plausibilität dieser drei Hypothesen sind eine Reihe von Einwendungen nicht von der Hand zu weisen, wie spätestens aus der ,Antwort' von Kohl und Leisering (1982) deutlich wird. Der Haupteinwand gegen die einfache Ableitung unterschiedlicher Armutsgefahren aus der Arbeitsmarktsegmentierung liegt in der Tatsache, daß die Versorgungslage einer Person nicht nur vom Erwerbs- und Sozialeinkommen abhängt, sondern ganz zentral auch durch das ,Einkommen' bestimmt wird, das ihr auf dem Wege innerfamiliärer Umverteilung in der Familie zufließt oder das sie selbst auf diesem Wege den anderen Haushaltsmitgliedern gewährt (Kohl und Leisering 1982: 420f.). Dieser Verteilungsaspekt ist aber im Rahmen der dargestellten Thesen ganz offensichtlich ausgespart - Heinze et al. (1983: 165) setzen sogar Personen und Haushalte $2 \mathrm{mal}$ durch einen Schrägstrich explizit gleich!

\section{Vorgehensweise, Operationalisierungen und Daten}

In dem vorliegenden Beitrag wird nachfolgend für die Bundesrepublik eine empirische Abwägung der konkurrierenden Einflüsse Arbeitsmarkt und Familie auf die individuelle Lebenslage von Erwerbstätigen und auf das Risiko des Abstiegs und der Verarmung durch Arbeitslosigkeit vorgenommen. Hierzu wird zunächst die individuelle Lebenslage Erwerbstätiger in Abhängigkeit von Arbeitsmarkt- und Familieneinflüssen beleuchtet (Punkt 4.1). Anschließend wird das Verarmungsund Abstiegsrisiko im Fall von Arbeitslosigkeit unter dem Einfluß der Arbeitsmarktsegmentierung und des Familienzusammenhangs untersucht (Punkt 4.2). In einer abschließenden Analyse wird schließlich das Risiko der Arbeitslosigkeit und das der Verarmung im Fall von Arbeitslosigkeit gemeinsam betrachtet und damit der Frage nach der individuellen Verarmungsgefahr durch Arbeitslosigkeit in Form der Kumulation von unsicherer 
Arbeitsmarktstellung und schlechter Absicherung nachgegangen (Punkt 4.3).

Die Untersuchung bezieht sich nur auf diejenigen Erwerbspersonen, ${ }^{1}$ die aktuell oder potentiell von Arbeitslosigkeit bedroht sind, alle Arbeiter, Angestellten und aktuell Arbeitslosen also, während die Beamten aus der Analyse ausgeblendet bleiben. Der Untersuchung liegen dabei Mikrosimulationsberechnungen unter Berücksichtigung des Familienkontexts zugrunde. Bei der Erfassung des Einflusses, den die Arbeitsmarktstrukturierung und der Familienzusammenhang auf die Armut Erwerbstätiger ausübt (Punkt 4.1), wurde für die aktuell Arbeitslosen ein fiktives Erwerbseinkommen zugrunde gelegt. ${ }^{2}$ Zur Analyse des Verarmungs- und Abstiegsrisikos bei Arbeitslosigkeit (Punkt 4.2) wurde umgekehrt für die aktuell Erwerbstätigen ein fiktives Arbeitslosigkeitseinkommen auf der Grundlage von institutionellen Regelungen der sozialen Sicherung simuliert. Diese Berechnung geht von dem Gedanken aus, daß Armut dann als Folge von Arbeitslosigkeit angesehen werden kann, wenn für dieselbe Person ohne Arbeitslosigkeit keine Armut bestünde und mit anderen Worten erst im Zuge der Arbeitslosigkeit ein Abstieg und eventuell eine Verarmung entsteht. Der Einkommensausfall bei Arbeitslosigkeit beruht also bei den aktuell Erwerbstätigen auf einer Simulation des bei Arbeitslosigkeit zur Verfügung stehenden Einkommens und bei den aktuell Arbeitslosen auf der zuvor erwähnten Simulation des Erwerbseinkommens. Übersicht 1 gibt einen Überblick über die Berechnungen.

Für die Situation der Arbeitslosigkeit wurde einheitlich unterstellt, daß der Arbeitslose Arbeitslosengeld bekommt und eventuell bestehende Wohngeldansprüche ausschöpft. Bei Verheirateten wurde außerdem von der Inanspruchnahme des steuerlichen Ehegattensplittings ausgegangen. Der so berechnete Einkommensausfall bei Arbeitslosigkeit kann daher als Mindestausfall in der

\footnotetext{
1 Zu den Erwerbspersonen zählen die Erwerbstätigen und die Erwerbslosen.
}

${ }^{2}$ Die Simulation der fiktiven Erwerbseinkommen aktuell Arbeitsloser erfolgte auf der Basis von Humankapitalschätzungen. Diese Schätzungen sind mit erheblichen Unsicherheiten belastet, die sich jedoch kaum entscheidend auf die hier untersuchte Fage von Armut oder Nicht-Armut auswirken. Die Berechnungen können hier auch insofern akzeptiert werden, als der Datensatz aus dem Jahr 1981 nur 81 Fälle aktueller Arbeitslosigkeit umfaßt. ersten Zeit der Arbeitslosigkeit interpretiert werden. Eine Nichtberechtigung zu Arbeitslosengeld oder eine Nichtinanspruchnahme von zustehenden Wohngeldansprüchen ${ }^{3}$ lassen den Einkommensausfall noch größer werden, und insbesondere in der längeren Folge von Arbeitslosigkeit ist spätestens nach dem Auslaufen des Arbeitslosengeldanspruchs mit einem weiteren Abstieg zu rechnen. Trotzdem erlaubt auch diese vereinfachende Vorgehensweise, die Struktüren der Verarmung bei Arbeitslosigkeit nach arbeitsmarkt- und familienbezogenen Merkmalen zuverlässig zu erfassen. ${ }^{4}$

Über die Untersuchung der auf die Verarmung bei Arbeitslosigkeit zugeschnittenen Frage hinaus, inwieweit sich die Arbeitsmarktdualisierung auch in das System der sozialen Sicherung hinein fortsetzt, wird abschließend untersucht, inwieweit die Arbeitsmarktdualisierung auch in der Kumulation eines erhöhten Arbeitslosigkeitsrisikos und einer schlechten Absicherung bei Arbeitslosigkeit wiederzufinden ist, und ob, wie von Heinze et al. (1981) postuliert, erhöhtes Arbeitslosigkeitsrisiko und erhöhtes Verarmungsrisiko bei eingetretener Arbeitslosigkeit personell zusammentreffen. Die wie beschrieben erfaßten Verarmungs- und Abstiegsrisiken bei Arbeitslosigkeit werden zu diesem Zweck mit den entsprechenden unterschiedlichen Arbeitslosigkeitsrisiken dergestalt in Verbindung gebracht, daß Arbeitslosigkeit analog der Arbeitslosigkeitsrisiken simuliert wird (s. Punkt 4.3).

Als Armut wird hier ein Einkommen unter der Armutsgrenze und als Verarmung eine Einkommensreduzierung bis unter die Armutsgrenze defi-

\footnotetext{
${ }^{3}$ Das Wohngeld ist in der Bundesrepublik eine der wichtigsten einkommensabhängigen Sozialleistungen und kann deshalb bei Arbeitslosigkeit eine gewisse Auffangfunktion erfüllen. Es gibt allerdings zahlreiche Indizien, daß das Wohngeld - ähnlich wie bei der Sozialhilfe seit langem bekannt (Hartmann 1981) - eine nicht unbeträchtliche Dunkelziffer der Nichtinanspruchnahme trotz Berechtigung aufweist. Da nicht gesagt werden kann, wer im Fall von Arbeitslosigkeit neu entstehendes oder höher ausfallendes Wohngeld in Anspruch nehmen wird, ist die Simulation auf die Annahme genereller Inanspruchnahme des jeweiligen Wohngeldanspruchs angewiesen.
}

${ }^{4}$ Alternative Annahmen über den Bezug von Arbeitslosengeld, Arbeitslosenhilfe und Wohngeld führen teilweise zu einem weit höheren Ausmaß der Verarmung bei Arbeitslosigkeit, führen aber zu nur unwesentlichen strukturellen Veränderungen des Zusammenhangs zwischen Arbeitsmarktsegmentierung bzw. Familienkontext und Verarmung (Klein 1987). 
niert. Das armutsabgrenzende Einkommensniveau wurde bei der Sozialhilfeschwelle gezogen. Ohne an dieser Stelle auf alle Einzelheiten der in den Berechnungen nachmodellierten Sozialhilfeschwelle einzugehen (s. hierzu auch Klein 1987), sei jedoch hier darauf hingewiesen, daß der armutsabgrenzende Einkommensbetrag in der Sozialhilfe familiengrößenabhängig gestaffelt ist. Bei dieser Staffelung sind auch altersabhängige $\mathrm{Be}$ darfsunterschiede der einzelnen Familienmitglieder und die Einsparungen durch gemeinsames Wirtschaften berücksichtigt (Klein 1986).

Zur Operationalisierung von Verarmung und Abstieg wird nachfolgend auf unterschiedliche Maße zurückgegriffen. Dies ist zunächst die Armutsquote, die den Anteil derjenigen in Armut an der Gesamtzahl der jeweils analysierten Erwerbspersonen wiedergibt. Demgegenüber bezieht sich die Verarmungsquote auf den Anteil der erst durch Arbeitslosigkeit armen Personen an der bei Erwerbstätigkeit nicht armen Erwerbsbevölkerung. Zur ergänzenden Analyse des sozialen Abstiegs, der noch über der Armutsgrenze endet, werden zwei weitere Maßzahlen herangezogen. Das wohlstands- oder bedarfsgewichtete Einkommen setzt das Familieneinkommen mit einem Gewicht für den familiengrößenabhängigen Bedarf in Beziehung, welches in Anlehnung an die Sozialhilferegelungen die Einsparungen durch gemeinsames Wirtschaften und individuelle Bedarfsunterschiede berücksichtigt. Das wohlstandsgewichtete Einkommen kann mithin als eine standardisierte Einkommensgröße verstanden werden, die einheitlich auf den Bedarf eines Einpersonenhaushalts hin standardisiert ist. Als Wohlstandsposition ist schließlich das Einkommen in Relation zur familiengrößenspezifischen Armutsgrenze definiert. Im Unterschied zu dem wohlstandsgewichteten Einkommen finden hierbei auch unterschiedliche Wohnungskosten Berücksichtigung. Die Wohlstandsposition gibt mit anderen Worten das Vielfache wieder, um das das Einkommen über der Armutsschwelle liegt.

Hinsichtlich des Arbeitsmarkteinflusses werden nachfolgend die Auswirkungen der Merkmale Ausbildung, Alter und Geschlecht auf die soziale Lage bei Erwerbstätigkeit und bei Arbeitslosigkeit analysiert. Nach der Segmentierungstheorie sind Lohnhöhe, Beschäftigungsstabilität, Arbeitsbedingungen und gewerkschaftlicher Organisationsgrad anhand dieser Merkmale verteilt (Heinze et al. 1981: 228f.). Das Segmentierungskonzept gibt jedoch keine konkreten Hinweise, wie aus den ge- nannten Merkmalen eine konkrete Operationalisierung der Arbeitsmarktsegmentzugehörigkeit abzuleiten ist. Auch Heinze et al. (1981) lassen diese Frage weitgehend offen. In Übereinstimmung mit unterschiedlichen Arbeitsmarktheorien kann jedoch davon ausgegangen werden, daß jedenfalls mit den genannten Merkmalen wichtige Arbeitsmarkteinflüsse erfaßt werden, die auch zur Abgrenzung der Problemgruppen auf dem Arbeitsmarkt beitragen.

Hinsichtlich der familialen Einflüsse auf die individuelle soziale Lage erscheinen vor allem drei Faktoren von Bedeutung: der Bedarf der Familie, der hauptsächlich durch die Familiengröße bestimmt wird, der Verdienerstatus sowie die Zahl und die Höhe weiterer Einkommen in der Familie. Um den Einfluß dieser Faktoren zu erfassen, werden nachfolgend die Lebenslage Erwerbstätiger und die Verarmungsgefahr Arbeitsloser auch in Abhängigkeit von Familiengröße, der Zahl der Verdienste in der Familie und, bei Arbeitslosen, dem Verdienerstatus als Haupt- oder nur Zweitverdiener analysiert.

Als Datengrundlage diente die sog. Einkommensund Transferumfrage des Sonderforschungsbereichs 3 der Universitäten Frankfurt und Mannheim von 1981. Die Umfrage umfaßt knapp 3000 Haushalte/Familien mit deutschem Haushaltsvorstand, in denen etwa 8000 Personen leben, und wurde anhand von Mikrozensusergebnissen repräsentativ auf die gesamte Bundesrepublik hochgerechnet. Die Einkommens- und Transferumfrage erfaßt alle personen- und familienbezogenen Einkommen im Haushaltszusammenhang und stellt deshalb für die hier berichtete Untersuchung eine ideale Mikrodatenbasis dar. Obwohl die Daten aus dem Jahr 1981 datieren, ist trotz der inzwischen höheren Arbeitslosigkeit mit wesentlichen Strukturverschiebungen bis heute nicht zu rechnen.

\section{Empirische Mikrosimulationsergebnisse zum Zusammenhang von Armut und Arbeitsmarkt}

\subsection{Die Armut Erwerbstätiger}

Um den Einfluß des Arbeitsmarkts und des Familienzusammenhangs auf die soziale Lage zu erfassen, sind in den Tabellen 1 bis 3 die Armutsquoten, das wohlstandsgewichtete Einkommen und die Wohlstandsposition nach den Merkmalen des Familienkontexts und der Arbeitsmarktstellung aufgeschlüsselt. Tabelle 1 bezieht sich zunächst nur 
Ubersicht 1 Berechnungen der Arbeitslosigkeitssimulation.

Variable Fall Berechnung

0. Aktuelles Haushaltseinkommen

I. Haushaltseinkommen bei Erwerbstätigkeit aller Enwerbspersonen im Haushalt (Referenzeinkommen)
1) alle Erwerbsp. sind erwerbstätig

2) zmdst. 1 Erwerbsp. ist arbeitslos mit ALU

3) Haushalte mit arbeitslosen Erwerbspersonen nur ohne ALU

1) Haupt-, Zweitverdiener und weitere Personen sind aktuell arbeitslos

2) Haupt-, Zweitverd. und mind. 1 weit. AhMitglied sind aktuell arbeitslos

3) nur Haupt-, Zweitverd. ist aktuell arbeitslos aktuelles Haushalts-Nettoeink. einschließlich Mietwert

aktuelles Haushalts-Nettoeink. einschl. Mietwert ohne Sozialhilfe

aktuelles Haush.-Nettoeink. wie unter I.1)

- ALU (tatsächlich)

- Splittingeffekt (errechnet)

- Wohngeld(-verminderung) (tats./err.)

+ Netto-Erw.-Eink. gem. ALU-Betrag resp. Humankapitalschätzung (errechnet)

aktuelles Haushalts-Nettoeink. wie unter I.1)

- Splittingeffekt (errechnet)

- Wohngeld(-verminderung) (tats./err.)

+ Nettoerwerbseink. gem. Humankapitalschätzung (errechnet)

Haush.-Nettoeink. wie unter I.

- Hauptverd.- (Zweitverd.-) Eink. (tats.)

+ ALG (errechnet)

+ Splittingeffekt (errechnet)

+ Wohngeld(-vermehrung) (err.)

Haush.-Nettoeink. wie unter I.

- Hauptverd.- (Zweitverd.-) Eink. (err.)

+ ALG d. Hauptverd. (tats., ggf. err.)

+ Wohngeld (errechnet)

aktuelles Haushalts-Nettoeink. einschl. Mietwert ohne SH

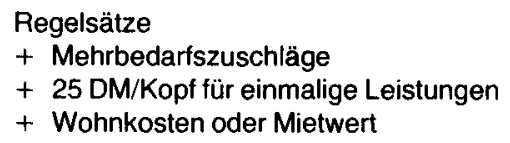

auf den Familienkontext. In Tabelle 1 finden die Familiengröße, der Verdienerstatus und die Verdienerzahl in der Familie Berücksichtigung. Die Berechnungen der Tabelle 1 gehen im übrigen davon aus, daß alle Erwerbspersonen im Haushalt erwerbstätig sind. In den in der Transferumfrage noch selteneren Arbeitslosigkeitsfällen wurde deshalb auf der Basis von Humankapitalschätzungen ein fiktives Erwerbseinkommen unterstellt. Diese Schätzung ist zwar recht unsicher; die in Tabelle 1 wiedergegebenen Werte sind jedoch gegenüber diesen Unsicherheiten sehr robust, da die Anzahl dieser Schätzungen relativ niedrig liegt und da es bezogen auf die Armutsquote - gar nicht auf den exakten Wert des fiktiven Erwerbseinkommens ankommt, sofern der Unsicherheitsbereich nicht bis zur Armutsschwelle hinunter reicht.
Bei Erwerbstätigkeit fallen also $3,8 \%$ der betrachteten Arbeiter, Angestellten und aktuell Arbeitslosen unter die Sozialhilfeschwelle (Tabelle 1, Spalte 1). Diese Messung beruht - wie gesagt nicht nur auf dem individuellen Verdienst, sondern auch auf der Haushaltsgröße und der Höhe weiterer Einkommen im Haushalt. Dem gemäß findet sich in Tabelle 1 (Spalte 1) eine sehr deutliche Differenzierung der Armutsquote nach der Familien- bzw. Haushaltsgröße. Die Alleinlebenden sind mit durchschnittlich 3,0\% einer leicht unterdurchschnittlichen Armutsquote ausgesetzt. Am niedrigsten ist die Armutsquote mit $1,8 \%$ unter den Arbeitern und Angestellten in Zweipersonenhaushalten. Diejenigen in größeren Haushalten sind mit zunehmender Haushaltsgröße zunehmend stärker von Armut betroffen; vor allem die Haus- 
halte mit fünf oder mehr Personen leben weit überdurchschnittlich häufig unter der Armutsschwelle (Tabelle 1).

Bezieht man sich in Tabelle 1 nur auf diejenigen, die in ihren Familien den Hauptverdienerstatus innehaben, so liegt die Armutsquote mit 4,1\% geringfügig über dem Gesamtdurchschnitt. Auch ein Alleinverdiener ist natürlich Hauptverdiener. Die höhere Armutsquote unter denjenigen Arbeitern und Angestellten, die zum Teil auch Alleinverdiener sind, kann deshalb als Anzeichen dafür gewertet werden, daß die Existenz eines Zweitver-

Tabelle 1 Armut und Wohlstand von Arbeitern und Angestellten nach der Haushaltsgröße, dem Verdienerstatus und der Verdienerzahl.

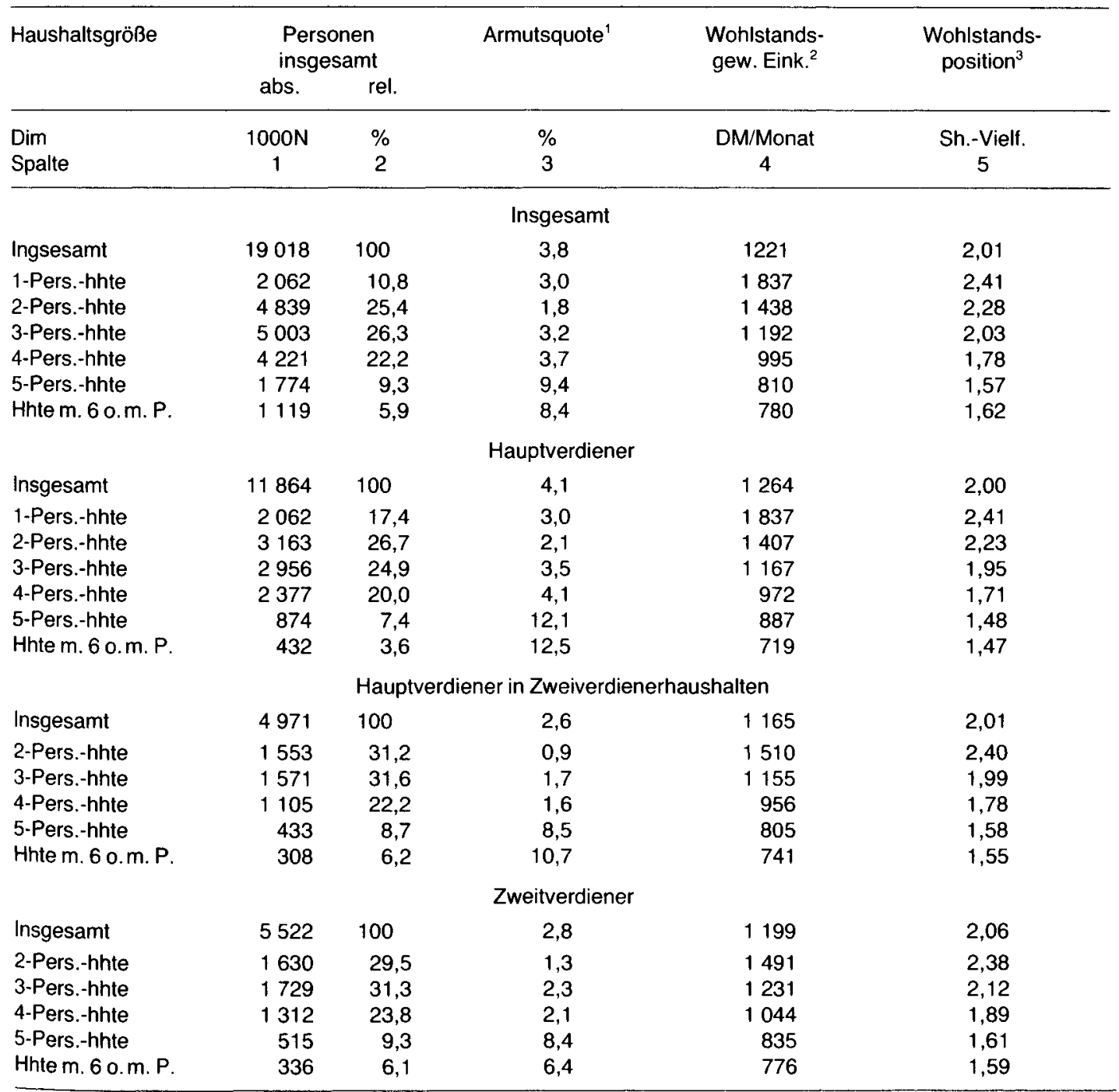

1 Die Armutsquote gibt den Anteil der Armutsbevölkerung in \% wieder.

${ }^{2}$ bezogen auf den Bedart eines Alleinstehenden

${ }^{3}$ in Vielfachen der Sozialhilfeschwelle

Quelle: Transferumfrage 1981; eigene Berechnungen 
dieners tendenziell vor Erwerbstätigenarmut schützt. Unter den Hauptverdienern findet sich ansonsten dieselbe $\mathbf{J}$-förmige Haushaltsgrößenstruktur der Armutsquoten.

Konzentriert man sich allerdings unter den Hauptverdienern auf diejenigen, in deren Familien noch ein zweiter Verdiener zum Familieneinkommen beiträgt (s. ebenfalls Tabelle 1), ist eine mit nur 2,6\% deutlich niedrigere Armutsquote festzustellen; die Haushaltsgrößenstruktur der Armutsquoten ist jedoch auch in dieser Gruppe ähnlich. Konzentriert man sich hingegen auf diejenigen Arbei-

Tabelie 2 Armut und Wohlstand von Arbeitern und Angestellten nach Alter, Geschlecht und Haushaltsgröße.

\begin{tabular}{|c|c|c|c|c|c|c|c|c|c|c|}
\hline \multirow{2}{*}{$\begin{array}{l}\text { Alter } \\
\text { in Jahren }\end{array}$} & \multicolumn{5}{|c|}{ Personen insgesamt } & \multicolumn{5}{|c|}{ Personen in 1-Personen-Haushalten } \\
\hline & abs. & rel. & $\begin{array}{l}\text { Armuts- } \\
\text { quote' }\end{array}$ & $\begin{array}{l}\text { Wohlfahrts- } \\
\text { gew. Eink. }\end{array}$ & $\begin{array}{l}\text { Wohlfahrts- } \\
\text { position }\end{array}$ & abs. & rel. & $\begin{array}{l}\text { Armuts- } \\
\text { quote }\end{array}$ & $\begin{array}{l}\text { Wohlfahrts- } \\
\text { gew. Eink. }\end{array}$ & $\begin{array}{l}\text { Wohlfahrts- } \\
{ }^{2} \text { position }^{3}\end{array}$ \\
\hline $\begin{array}{l}\text { Dim } \\
\text { Spalte }\end{array}$ & $\begin{array}{c}1000 \mathrm{~N} \\
1\end{array}$ & $\begin{array}{l}\% \\
2\end{array}$ & $\begin{array}{l}\% \\
3\end{array}$ & $\begin{array}{l}\text { DM/Monat } \\
4\end{array}$ & $\begin{array}{l}\text { Sh.-Vielf. } \\
5\end{array}$ & $\begin{array}{c}1000 \mathrm{~N} \\
6\end{array}$ & $\begin{array}{l}\% \\
7\end{array}$ & $\begin{array}{l}\% \\
8\end{array}$ & $\begin{array}{l}\text { DM/Monat } \\
\quad 9\end{array}$ & $\begin{array}{l}\text { Sh.-Vielf. } \\
10\end{array}$ \\
\hline Insgesamt & 19018 & 100 & 3,8 & 1221 & 2,01 & 2062 & 100 & 3,0 & 1837 & 2,41 \\
\hline $\begin{array}{r}-25 \\
26-30 \\
31-35\end{array}$ & $\begin{array}{l}4973 \\
2116 \\
1529\end{array}$ & $\begin{array}{r}26,1 \\
11,1 \\
8,0\end{array}$ & $\begin{array}{l}5,7 \\
1,5 \\
0,8\end{array}$ & $\begin{array}{l}1051 \\
1355 \\
1341\end{array}$ & $\begin{array}{l}1,89 \\
2,15 \\
2,10\end{array}$ & $\begin{array}{l}500 \\
497\end{array}$ & $\begin{array}{l}24,3 \\
24,1\end{array}$ & $\begin{array}{l}4,2 \\
0,0\end{array}$ & $\begin{array}{l}1469 \\
1894\end{array}$ & $\begin{array}{l}2,13 \\
3,43\end{array}$ \\
\hline $\begin{array}{l}36-40 \\
41-45\end{array}$ & $\begin{array}{l}2305 \\
2496\end{array}$ & $\begin{array}{l}12,1 \\
13,1\end{array}$ & $\begin{array}{l}5,0 \\
3,3\end{array}$ & $\begin{array}{l}1264 \\
1195\end{array}$ & $\begin{array}{l}1,98 \\
1,97\end{array}$ & 405 & 19,6 & 5,7 & 2099 & 2,60 \\
\hline $\begin{array}{l}46-50 \\
51-55\end{array}$ & $\begin{array}{l}2048 \\
1781\end{array}$ & $\begin{array}{r}10,8 \\
9,4\end{array}$ & $\begin{array}{l}2,8 \\
4,3\end{array}$ & $\begin{array}{l}1188 \\
1271\end{array}$ & $\begin{array}{l}1,98 \\
2,07\end{array}$ & & & & & \\
\hline $\begin{array}{l}56-60 \\
61-65 \\
66 \text { u.m. }\end{array}$ & $\begin{array}{r}1272 \\
243 \\
255\end{array}$ & $\begin{array}{l}6,7 \\
1,3 \\
1,3\end{array}$ & $\begin{array}{r}1,6 \\
2,9 \\
13,0\end{array}$ & $\begin{array}{ll}1 & 418 \\
1 & 487 \\
1 & 216\end{array}$ & $\begin{array}{l}2,19 \\
2,25 \\
1,92\end{array}$ & 660 & 32,0 & 2,6 & 1912 & 2,48 \\
\hline Männer & 11542 & 100 & 3,3 & 1200 & 1,99 & 977 & 100 & 2,2 & 1984 & 2,60 \\
\hline $\begin{array}{r}-25 \\
26-30 \\
31-35\end{array}$ & $\begin{array}{l}2635 \\
1248 \\
1018\end{array}$ & $\begin{array}{r}22,8 \\
10,8 \\
8,8\end{array}$ & $\begin{array}{l}4,5 \\
2,5 \\
1,2\end{array}$ & $\begin{array}{l}1015 \\
1322 \\
1302\end{array}$ & $\begin{array}{l}1,86 \\
2,07 \\
2,04\end{array}$ & $\begin{array}{l}202 \\
284\end{array}$ & $\begin{array}{l}21,4 \\
30,0\end{array}$ & $\begin{array}{r}10,5 \\
0,0\end{array}$ & $\begin{array}{l}1524 \\
2086\end{array}$ & $\begin{array}{l}2,28 \\
2,54\end{array}$ \\
\hline $\begin{array}{l}36-40 \\
41-45\end{array}$ & $\begin{array}{l}1441 \\
1558\end{array}$ & $\begin{array}{l}12,5 \\
13,5\end{array}$ & $\begin{array}{l}5,5 \\
2,0\end{array}$ & $\begin{array}{l}1302 \\
1189\end{array}$ & $\begin{array}{l}2,00 \\
1,94\end{array}$ & 284 & 30,0 & 0,0 & 2232 & 2,78 \\
\hline $\begin{array}{l}46-50 \\
51-55\end{array}$ & $\begin{array}{l}1333 \\
1156\end{array}$ & $\begin{array}{l}11,5 \\
10,0\end{array}$ & $\begin{array}{l}2,8 \\
1,5\end{array}$ & $\begin{array}{l}1086 \\
1296\end{array}$ & $\begin{array}{l}1,92 \\
2,13\end{array}$ & & & & & \\
\hline $\begin{array}{l}56-60 \\
61-65 \\
66 \text { u. m. }\end{array}$ & $\begin{array}{l}797 \\
208 \\
162\end{array}$ & $\begin{array}{l}6,9 \\
1,8 \\
1,4\end{array}$ & $\begin{array}{r}2,6 \\
3,3 \\
16,4\end{array}$ & $\begin{array}{ll}1 & 315 \\
1 & 185 \\
1 & 164\end{array}$ & $\begin{array}{l}2,13 \\
1,95 \\
1,84\end{array}$ & 176 & 18,6 & 0.0 & 1990 & 2,87 \\
\hline Frauen & 7476 & 100 & 4,5 & 1254 & 2,04 & 1085 & 100 & 3,7 & 1705 & 2,23 \\
\hline $\begin{array}{r}-25 \\
26-30 \\
31-35\end{array}$ & $\begin{array}{r}2338 \\
868 \\
511\end{array}$ & $\begin{array}{r}31,3 \\
11,6 \\
6,8\end{array}$ & $\begin{array}{l}7,1 \\
0,0 \\
0,0\end{array}$ & $\begin{array}{l}1092 \\
1403 \\
1419\end{array}$ & $\begin{array}{l}1,92 \\
2,26 \\
2,21\end{array}$ & $\begin{array}{l}298 \\
213\end{array}$ & $\begin{array}{l}26,7 \\
19,1\end{array}$ & $\begin{array}{l}0,0 \\
0,0\end{array}$ & $\begin{array}{l}1431 \\
1639\end{array}$ & $\begin{array}{l}2,03 \\
2,28\end{array}$ \\
\hline $\begin{array}{l}36-40 \\
41-45\end{array}$ & $\begin{array}{l}864 \\
938\end{array}$ & $\begin{array}{l}11,6 \\
12,5\end{array}$ & $\begin{array}{l}4,1 \\
5,5\end{array}$ & $\begin{array}{l}1201 \\
1205\end{array}$ & $\begin{array}{l}1,95 \\
2,02\end{array}$ & (121) & $(10,8)$ & $(19,0)$ & 1788 & 2,17 \\
\hline $\begin{array}{l}46-50 \\
51-55\end{array}$ & $\begin{array}{l}715 \\
625\end{array}$ & $\begin{array}{l}9,6 \\
8,4\end{array}$ & $\begin{array}{l}2,8 \\
9,4\end{array}$ & $\begin{array}{l}1379 \\
1225\end{array}$ & $\begin{array}{l}2,10 \\
1,96\end{array}$ & & & & & \\
\hline $\begin{array}{l}56-60 \\
61-65 \\
66 \text { u. m. }\end{array}$ & $\begin{array}{l}475 \\
/ \\
(93)\end{array}$ & $\begin{array}{l}6,3 \\
0,7 \\
1,2\end{array}$ & $\begin{array}{l}0,0 \\
1 \\
(7,0)\end{array}$ & $\begin{array}{c}1591 \\
/ \\
(1307)\end{array}$ & $\begin{array}{c}2,29 \\
/ \\
(2,06)\end{array}$ & 484 & 43,3 & 3,6 & 1884 & 2,34 \\
\hline
\end{tabular}

${ }^{1}$ Die Armutsquote gibt den Anteil der Armutsbevölkerung in Prozent wieder.

${ }^{2}$ bezogen auf den Bedart eines Alleinlebenden

3 in Vielfachen der Sozialhilfeschwelle 
ter und Angestellten, die in ihren Familien einen Zweitverdienerstatus innehaben, ist die durchschnittliche Armutsquote mit 2,8\% eher etwas höher, aber immer noch deutlich unter dem Gesamtdurchschnitt von $3,8 \%$.

Es scheint sich damit folgende Abhängigkeit der Erwerbstätigenarmut von der Familiengröße herauszukristallisieren. Je größer die Familie, um so höher ist die Armutsquote, wobei vor allem beim Übergang vom Vier- zum Fünfpersonenhaushalt ein deutlicher Sprung zu vermerken ist. Ein zweiter Verdiener wirkt sich deutlich armutsverringernd aus. In der Gesamtheit aller Arbeiter und Angestellten münden beide Zusammenhänge in eine J-förmige Haushaltsgrößenstruktur der Erwerbstätigenarmut. Der Verdienerstatus als Erstoder Zweitverdiener ist jedoch - bezogen auf die Haushalte, in denen mindestens zwei Verdiener zum Familieneinkommen beitragen - weitgehend unerheblich für die Frage nach der Erwerbstätigenarmut.

Betrachtet man das mehr generelle Wohlstandsniveau, so lebt der Durchschnitt der Arbeiter- und Angestelltenschaft 1981 auf einem (wohlstandsbereinigten) Einkommensniveau von $1221 \mathrm{DM}$ bzw. dem 2,01fachen der Sozialhilfeschwelle. Mit zunehmender Haushaltsgröße ist durchgehend ein abnehmendes Wohlstandsniveau festzustellen (Tabelle 1). Verdienerzahl und Verdienerstatus erscheinen relativ irrelevant. Dies ist, besonders was die Verdienerzahl anbetrifft, erstaunlich. Die Zweitverdienste konzentrieren sich offenbar in den ohne den Zweitverdienst eher schlechter gestellten Familien (s. a. Klein 1985).

Tabelle 2 bezieht sich auf die Arbeitsmarktvariablen Alter und Geschlecht. Im linken Teil von Tabelle 2 ist zunächst in der Gesamtheit der Arbeiter- und Angestelltenschaft keine einheitliche Altersdifferenzierung der Armutsquote und des durchschnittlichen Wohlstandsniveaus zu beobachten. wenngleich die Armutsquoten mit Werten zwischen 0,8 und $13,0 \%$ doch eine beträchtliche Variationsbreite anzeigen. Frauen sind allerdings im Einklang mit der Theorie des segmentierten Arbeitsmarkts mit einer Verarmungsquote von $4.5 \%$ deutlich überdurchschnittlich von Erwerbstätigenarmut betroffen. Hinsichtlich des durchschnittlichen Wohlstandsniveaus sind aber Frauen eher etwas besser gestellt. Wenn man berücksichtigt, daß Frauen im Durchschnitt niedrigere Löhne beziehen, ist dieses letztere Ergebnis nur mit den Einflüssen des Familienzusammenhangs zu erklä- ren, damit zum Beispiel, daß erwerbstätige Frauen in aller Regel in Familien leben, in denen noch ein zweiter Verdiener erwerbstätig ist, während erwerbstätige Männer häufig den Alleinverdiener stellen.

Auch aus der kombinierten Betrachtung von Alters- und Geschlechtseinflüssen auf die Armutsquote läßt sich nicht ohne weiteres eine Evidenz für erhöhte Erwerbstätigenarmut im zweiten Arbeitsmarktsegment ableiten. Unter den Frauen weist zwar die jüngste Altersgruppe mit $7,1 \%$ eine weit überdurchschnittliche Armutsquote auf; ansonsten sind aber eher die mittleren Altersgruppen von Erwerbstätigenarmut betroffen. Unter den Männern läßt sich hingegen gar keine einheitliche Altersstrukturierung der Armutsquoten feststellen. Nur das Geschlecht scheint somit im Sinne der Segmentierungstheorie einen Einfluß auf die Verteilung der Erwerbstätigenarmut auszuüben.

Es stellt sich jedoch die Frage, inwieweit Arbeitsmarkteinflüsse durch unterschiedliche Familienzusammenhänge lediglich überlagert werden. Angesichts der zuvor aufgezeigten Differenzierung der Erwerbstätigenarmut nach dem Familienkontext ist deshalb im rechten Teil von Tabelle 2 nur auf Alleinstehende Bezug genommen worden. Hier zeigt sich ein deutlicher Alters- und Geschlechtseinfluß auf die Armutsquote und auf das generelle Wohlstandsniveau, der zuvor offensichtlich durch den Einfluß unterschiedlicher Familienzusammenhänge überlagert war. Die geschlechtsspezifischen Armutsquoten wie auch die geschlechtsspezifischen Unterschiede der Wohlstandsposition zeigen in den Einpersonenhaushalten eine noch ausgeprägtere Besserstellung der Männer an. Unter den Männern sind allerdings nur die jüngeren und unter den Frauen nur die älteren von Erwerbstätigenarmut betroffen (Tabelle 2, Spalte 8). Auch hinsichtlich des Alters scheint sich damit - wenn der Familienzusammenhang ausgeblendet wird - eine Benachteiligung des zweiten Segments in Form einer erhöhten Armutsbetroffenheit bei Jugendlichen und Älteren zu bestätigen. Der durchschnittliche Wohlstand zeigt allerdings eine mit zunehmendem Alter durchgehend steigende Tendenz. ${ }^{5}$

\footnotetext{
5 Das wohlstandsgewichtete Einkommen und die Wohlstandsposition in Vielfachen der Armutsgrenze zeigen u. U. etwas unterschiedliche Ergebnisse, weil die Armutsgrenze entsprechend der Sozialhilferegelung auch durch die Wohnkosten mitbestimmt wird.
} 
Tabelle 3 Erwerbstätigen-Armut und -Wohlstand von Arbeitern und Angestellten nach dem beruflichen Ausbildungsabschluß.

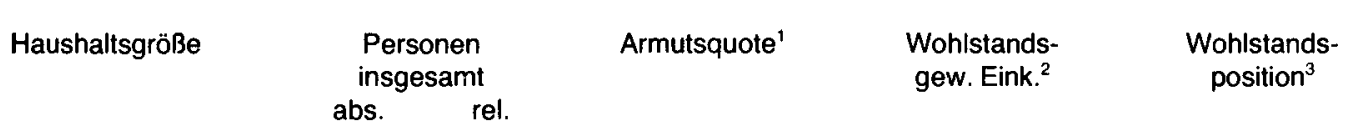

\begin{tabular}{lccccc}
\hline Dim & & & & DM/Monat & Sh. Vielf. \\
Spalte & $1000 \mathrm{~N}$ & $\%$ & $\%$ & 4 & 5
\end{tabular}

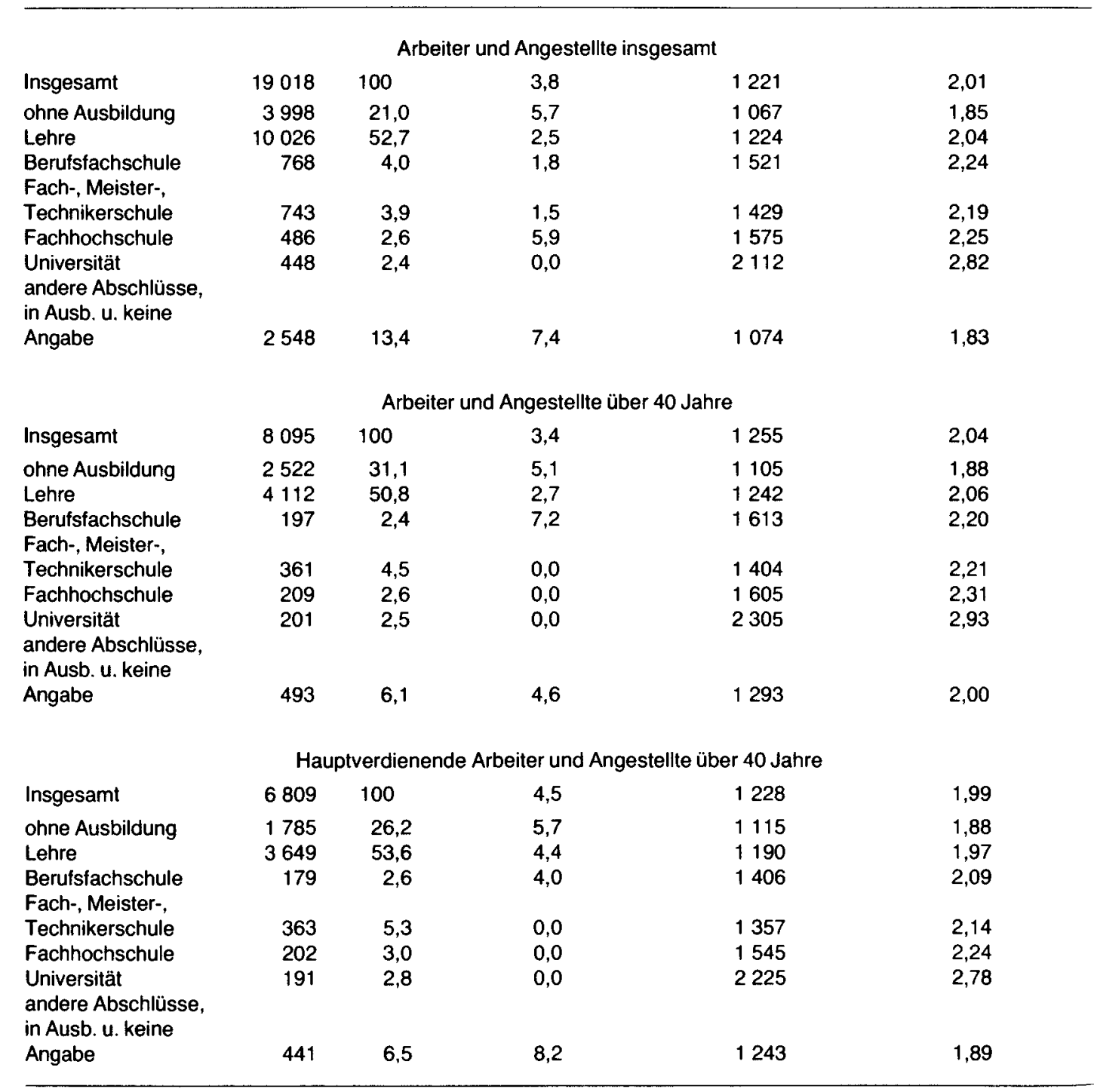

' Die Armutsquote gibt den Anteil der Armutsbevölkerung in Prozent wieder.

${ }^{2}$ bezogen auf den Bedarf eines Alleinlebenden

${ }^{3}$ in Vielfachen der Sozialhilfeschwelle

Quelle: Transferumfrage 1981; eigene Berechnungen 
In Tabelle 3 sind schließlich Erwerbstätigenarmut und Wohlstand nach dem beruflichen Ausbildungsabschlu 3 aufgegliedert. ${ }^{6}$ Eine nicht unbeträchtliche Anzahl von 13,4\% der Arbeiter- und Angestelltenschaft konnte in die aufgelisteten Ausbildungskategorien nicht eingeordnet werden, weil anderweitige Ausbildungsgänge absolviert worden waren, weil die Ausbildung noch nicht abgeschlossen war oder weil aus sonstigen Gründen keine Angabe vorliegt. Technische und gewerbliche Lehrabschlüsse wurden wegen der ähnlichen Ergebnisse nicht unterschieden. Hauswirtschaftliche Lehrabschlüsse waren im Datensatz so selten vertreten, daß für diese Gruppe keine speziellen Aussagen gesichert getroffen werden konnten. Im oberen Teil von Tabelle 3 ist in der Gesamtheit der Arbeiter- und Angestelltenschaft ein eher uneinheitlicher Zusammenhang zwischen dem Ausbildungsabschluß und der Betroffenheit von Erwerbstätigenarmut zu beobachten. Mit am stärksten betroffen sind diejenigen ohne Ausbildungsabschluß, während die Universitätsabsolventen bei Erwerbstätigkeit überhaupt nicht von Armut betroffen sind. Dazwischen weisen jedoch auch die Fachhochschulabsolventen eine überdurchschnittliche Betroffenheit auf. Wie aus dem mittleren Teil von Tabelle 3, der sich nur auf diejenigen über 40 Jahre bezieht, ersichtlich, konzentriert sich die überdurchschnittliche Betroffenheit der Fachhochschulabsolventen auf die jüngeren Altersgruppen unter 40 . Unter den älteren fallen jedoch die Berufsfachschulabsolventen durch eine hohe Armutsquote von 7,2\% aus dem Rahmen. Erst im unteren Teil von Tabelle 3, in dem neben dem Alter (über 40 Jahre) auch die Verdienerstellung im Haushalt durch Konzentration auf den Hauptverdiener vereinheitlicht wurde, ist eine klare Ausbildungsdifferenzierung der Erwerbstätigenarmut im Sinne der Theorie des dualen Arbeitsmarkts auszumachen. ${ }^{6}$ Mit zunehmendem Ausbildungsabschluß sinkt offenbar unter den Hauptverdienern über 40 die Armutsbetroffenheit. Ein deutlicher Niveauunterschied, der auch als Grenze zwischen den Arbeitsmarktsegmenten interpretiert werden könnte, liegt zwischen den Berufsfachschulabsolventen und denjenigen mit Fach-, Meister- oder Technikerabschluß. Der Ausbildungsabschluß kann damit am ehesten als armutsdifferenzierend angesehen werden.

"Eine Ausgliederung der Alleinlebenden war hier wegen zu geringer Fallzahien nicht möglich.
Auch für die Verteilung der Erwerbstätigenarmut nach dem Ausbildungsabschluß ist jedoch der Familienkontext, vor allem die Verdienerstellung in der Familie, offensichtlich nicht ohne Bedeutung.

\subsection{Verarmung bei Arbeitslosigkeit}

In den nun folgenden Analysen wird zunächst Arbeitslosigkeit unterstellt, unabhängig davon, ob sie aktuell zum Zeitpunkt der Befragung eingetreten und wie hoch ihre Gefahr einzuschätzen war. Die Analyse geht also nunmehr der Frage nach, inwieweit sich eine Arbeitsmarktbenachteiligung auch bei Arbeitslosigkeit in das System der sozialen Sicherung hinein fortsetzt. Diese Fortsetzung dürfte am deutlichsten zutage treten, wenn man wie in diesem Abschnitt unterschiedliche Arbeitslosigkeitsrisiken zunächst außer acht läßt. ${ }^{7}$ Die Verteilung der Sicherungswirkungen nach arbeitsmarktund familienbezogenen Merkmalen bezieht sich im Rahmen der hier berichteten Simulationsergebnisse hauptsächlich auf die Leistungsseite des sozialen Sicherungssystems, während die Verteilung von Anspruchsvoraussetzungen kaum untersucht werden konnte. Im Mittelpunkt der Analyse stehen daher unterschiedliche Sozialeinkommen aus denselben Sicherungssystemen, die teilweise an den früheren Arbeitsverdienst gekoppelt sind. Hinsichtlich des Zugangs zu einzelnen Systemen der sozialen Sicherung wurde hingegen generell von dem Bezug von Arbeitslosengeld und Wohngeld und der Inanspruchnahme steuerlicher Splittingvorteile ausgegangen. Soweit Anspruchs- und Leistungsunterschiede auf dieselbe Weise unterschiedlich verteilt sind, könnte eine gewisse Unterschätzung der Absicherungsunterschiede der Arbeitslosigkeit stattfinden. So wäre es beispielsweise vorstellbar, daß Arbeitnehmer aufgrund unstabilerer Beschäftigungsverhältnisse nicht lange genug sozialversicherungspflichtig beschäftigt waren, um bei Arbeitslosigkeit Arbeitslosengeld zu erhalten, so daß sie auf die niedrigere Arbeitslosenhilfe verwiesen wären. Nach wie vor hat jedoch die originäre, vom Beginn der Arbeitslosigkeit an gezahlte Arbeitslosenhilfe nur geringe Bedeutung. ${ }^{8}$ Die Be-

\footnotetext{
${ }^{7}$ Die Kombination unterschiedlicher Arbeitslosigkeitsrisiken und unterschiedlicher Verarmungsrisiken im Fall von Arbeitslosigkeit wird anschließend in Punkt 4.3 analysiert.
}

${ }^{8}$ Arbeitslosenhilfe wird i.d. R. erst nach Auslaufen des Arbeitslosengeldanspruchs bezogen. 
schränkung auf unterschiedliche Sozialleistungen ist deshalb, wenn man sich nur auf die erste Zeit der Arbeitslosigkeit bis zu maximal einem Jahr bezieht, durchaus vertretbar.

In Tabelle 4 ist die Gefahr von Verarmung und Abstieg zunächst wieder nach den Merkmalen des Familienzusammenhangs aufgeschlüsselt. Die Verarmungsquote, der Anteil derer also, die bei Ar- beitslosigkeit unter die Armutsschwelle absinken, beträgt im Gesamtdurchschnitt der Arbeiter- und Angestelltenschaft $9,9 \%$. Wie schon zuvor für die Erwerbstätigenarmut festgestellt, findet sich auch bei der Verarmung durch Arbeitslosigkeit eine annähernd J-förmige Haushaltsgrößenstruktur. Hinsichtlich des Verdienerstatus und der Verdienerzahl sind ebenfalls keine strukturellen Unter-

Tabelle 4 Verarmung und Abstieg im Fall von Arbeitslosigkeit nach der Haushaltsgröße, dem Verdienerstatus und der Verdienerzahl im Haushalt.

\begin{tabular}{|c|c|c|c|}
\hline Haushaltsgröße & Verarmungsquote $^{1}$ & $\begin{array}{l}\text { relativer Einkommens- } \\
\text { ausfall }^{2}\end{array}$ & $\begin{array}{l}\text { absoluter Wohlstands- } \\
\text { verlust }^{3}\end{array}$ \\
\hline $\begin{array}{l}\text { Dim } \\
\text { Spalte }\end{array}$ & $\begin{array}{c}\% \\
1\end{array}$ & $\begin{array}{l}\% \\
2\end{array}$ & $\begin{array}{c}\text { Sh.-Vielf. } \\
3\end{array}$ \\
\hline \multicolumn{4}{|c|}{ Insgesamt } \\
\hline Ingsesamt & 9,9 & 20,4 & 0,42 \\
\hline $\begin{array}{l}\text { 1-Pers.-hhte } \\
\text { 2-Pers.-hhte } \\
\text { 3-Pers.-hhte } \\
\text { 4-Pers.-hhte } \\
\text { 5-Pers.-hhte } \\
\text { Hhte m. } 6 \text { o. m.P. }\end{array}$ & $\begin{array}{r}10,8 \\
5,0 \\
7,9 \\
12,8 \\
19,6 \\
13,6\end{array}$ & $\begin{array}{l}29,4 \\
20,3 \\
19,9 \\
18,6 \\
19,6 \\
14,0\end{array}$ & $\begin{array}{l}0,71 \\
0,47 \\
0,39 \\
0,35 \\
0,30 \\
0,21\end{array}$ \\
\hline \multicolumn{4}{|c|}{ Hauptverdiener } \\
\hline Insgesamt & 11,1 & 23,5 & 0,49 \\
\hline $\begin{array}{l}\text { 1-Pers.-hhte } \\
\text { 2-Pers.-hhte } \\
\text { 3-Pers.-hhte } \\
\text { 4-Pers.-hhte } \\
\text { 5-Pers.-hhte } \\
\text { Hhte m. } 6 \text { o.m.P. }\end{array}$ & $\begin{array}{r}10,8 \\
5,3 \\
8,8 \\
16,1 \\
26,0 \\
19,1\end{array}$ & $\begin{array}{l}29,4 \\
22,6 \\
23,6 \\
22,2 \\
20,0 \\
14,4\end{array}$ & $\begin{array}{l}0,71 \\
0,52 \\
0,46 \\
0,41 \\
0,29 \\
0,21\end{array}$ \\
\hline \multicolumn{4}{|c|}{ Hauptverdiener in Zweiverdienerhaushalten } \\
\hline Insgesamt & 6,6 & 19,3 & 0,41 \\
\hline $\begin{array}{l}\text { 2-Pers.-hhte } \\
\text { 3-Pers.-hhte } \\
\text { 4-Pers.-hhte } \\
\text { 5-Pers.-hhte } \\
\text { Hhte m. } 6 \text { o. m.P. }\end{array}$ & $\begin{array}{r}0,9 \\
6,4 \\
11,3 \\
13,9 \\
11,5\end{array}$ & $\begin{array}{l}19,8 \\
20,9 \\
18,6 \\
18,4 \\
12,3\end{array}$ & $\begin{array}{l}0,50 \\
0,42 \\
0,38 \\
0,28 \\
0,20\end{array}$ \\
\hline \multicolumn{4}{|c|}{ Zweitverdiener } \\
\hline Insgesamt & 6,1 & 13,2 & 0,27 \\
\hline $\begin{array}{l}\text { 2-Pers.-hhte } \\
\text { 3-Pers.-hhte } \\
\text { 4-Pers.-hhte } \\
\text { 5-Pers.-hhte } \\
\text { Hhte m. } 6 \text { o. m.P. }\end{array}$ & $\begin{array}{r}3,9 \\
5,0 \\
7,7 \\
9,8 \\
11,5\end{array}$ & $\begin{array}{l}14,1 \\
12,4 \\
12,4 \\
14,9 \\
13,1\end{array}$ & $\begin{array}{l}0,33 \\
0,26 \\
0,24 \\
0,24 \\
0,20\end{array}$ \\
\hline
\end{tabular}

- Die Verarmungsquote gibt den Anteil der bei Arbeitsiosigkeit Verarmenden an den bei Erwerbstätigkeit nicht armen Arbeitern und Angestellten wieder.

2 in $\%$

3 in Vielfachen der Sozialhilfeschwelle

Quelle: Transferumfrage 1981; eigene Berechnungen 
schiede zur Erwerbstätigenarmut festzustellen. Auch für die sozialstaatliche Absicherung bei Arbeitslosigkeit gilt also: Größere Haushalte sind stärker verarmungsgefährdet als kleinere Haushalte. Bei mehreren Verdiensten in der Familie besteht ein wesentlich geringeres Verarmungsrisiko. Vergleichsweise unerheblich ist hingegen, ob der Arbeitslose den Haupt- oder ,nur' einen zweiten Verdiener in der Familie stellt.

Nicht uninteressant ist auch ein Blick auf den mehr generellen sozialen Abstieg. Wie aus Tabelle 4 ebenfalls zu entnehmen, ist der relative Einkommensausfall in drei Stufen mit der Haushaltsgröße verknüpft. Den größten relativen Abstieg vollziehen bei Arbeitslosigkeit die Alleinlebenden mit fast $30 \%$. Einen geringeren Abstieg erfahren diejenigen, die in Familien mit 2 bis 5 Personen leben. Der Einkommensausfall beträgt in diesen Haushalten im Gesamtdurchschnitt und bei den Hauptverdienern um die $20 \%$, bei den Zweitverdienern (einschließlich der Haushalte mit 6 oder mehr Personen) 12 bis $14 \%$. Diejenigen Arbeitslosen, die in Haushalten mit 6 oder mehr Personen leben, machen den geringsten relativen Abstieg durch. Der in Vielfachen der Armutsgrenze gemessene absolute Abstieg fällt mit zunehmender HaushaltsgröBe. Die Haushaltsgrößenstruktur des sozialen Abstiegs bei Arbeitslosigkeit unterscheidet sich damit kaum von der nach der Haushaltsgröße ungleichen Armutsbetroffenheit bei Erwerbstätigkeit. Die Verdienerzahl ist für den sozialen Abstieg bei Arbeitslosigkeit (wie schon für die Wohlstandsposition bei Erwerbstätigkeit festgestellt) von ebenfalls nur geringer Bedeutung. Zweitverdienerarbeitslosigkeit führt jedoch mit einem durchschnittlichen Abstieg von 13,2\% bzw. 0,27 Sozialhilfevielfachen zu einem deutlich geringeren Abstieg als Hauptverdienerarbeitslosigkeit (Tabelle 4).

Gegenüber der insoweit festgestellten Differenzierung des Abstiegs- und Verarmungsrisikos nach Merkmalen des familialen Lebenszusammenhangs läßt das Alter, das vor allem die Stellung auf dem Arbeitsmarkt beeinflußt, zunächst keinen systematischen Einfluß auf die Gefahr der Verarmung und das durchschnittliche Ausmaß des sozialen Abstiegs bei Arbeitslosigkeit erkennen (Tabelle 5, linker Teil). Hinsichtlich des Geschlechts sind Frauen mit einer Verarmungsquote von $7,1 \%$ und einem durchschnittlichen Abstieg von $16,8 \%$ weniger verarmungs- und abstiegsbedroht als Männer, ein Ergebnis, das sogar auf den ersten Blick den aus der Arbeitsmarktsegmentierung abgeleiteten Hypothesen entgegensteht. In der Verbindung von
Alter und Geschlecht zeigt sich jedoch in der Gesamtheit der jugendlichen Frauen bis 25 Jahre und der älteren Frauen ab 56 Jahre eine stark überdurchschnittliche Verarmungsgefahr bei Arbeitslosigkeit, gerade bei den Gruppen also, für die von Heinze et al. (1981) eine erhöhte Armutsgefahr abgeleitet wird. Auch der soziale Abstieg, der durch Arbeitslosigkeit ausgelöst wurde, scheint bei den Frauen eher auf die jungen und älteren konzentriert.

Die Arbeitsmarktdifferenzierung des Verarmungsrisikos nach Alter und Geschlecht tritt sehr viel deutlicher hervor, wenn man sich im rechten Teil von Tabelle 5 unter Vereinheitlichung unterschiedlicher Familiensituationen nur auf die Alleinstehenden beschränkt. Bezogen auf die Alleinlebenden, sind Frauen bei Arbeitslosigkeit mit 14,0 gegenüber $7,3 \%$ fast der doppelten Verarmungsgefahr ausgesetzt. Die zuvor für die Gesamtheit der Frauen festgestellte unterdurchschnittliche Verarmungsquote von $7,1 \%$ erscheint damit durch familiale ,Absicherung' bedingt. Die in Einpersonenhaushalten durchaus im Sinne der Segmentierungstheorie vorhandene Geschlechtsdifferenzierung des Verarmungsrisikos wird offenbar von einer unterschiedlichen familialen Absicherung von Männern und Frauen überlagert. Unter den Frauen sind - wiederum entsprechend der Arbeitsmarktsegmentierung - gerade die jüngeren und die älteren Frauen besonders verarmungsgefährdet. Bei den Männern konzentriert sich hingegen die Verarmungsgefahr bei Arbeitslosigkeit in den mittleren Altersgruppen zwischen 26 und 45 Jahren. Der allgemeine, auf der Grundlage der Theorie des segmentierten Arbeitsmarkts behauptete Geschlechtsunterschied ist also nur unter den $\mathrm{Al}$ leinlebenden ersichtlich, unter denen sich für Frauen fast die doppelte Verarmungsquote der Männer errechnet. In der Gesamtheit der Arbeiter- und Angestelltenschaft wird hingegen der arbeitsmarktbedingte Geschlechtsunterschied durch den Einfluß unterschiedlicher familialer Lebenszusammenhänge von Männern und Frauen überlagert, als dessen Resultat die Gesamtheit der Frauen sogar einer geringeren Verarmungsgefahr bei Arbeitslosigkeit ausgesetzt ist. Wenngleich somit die Arbeitsmarktmerkmale Alter und Geschlecht im Sinne der Segmentierungstheorie auch in die Arbeitslosigkeit fortwirken, wird doch der Einfluß der Arbeitsmarktsegmentierung gerade bei Arbeitslosen besonders stark durch den der Familienstruktur überlagert.

Von Heinze et al. (1981) wird schließlich in Anlehnung an die Theorie des segmentierten Arbeits- 
Tabelle 5 Verarmung und Abstieg im Fall von Arbeitslosigkeit nach Alter, Geschlecht und Haushaltsgröße.

\begin{tabular}{|c|c|c|c|c|c|c|}
\hline \multirow[b]{2}{*}{$\begin{array}{l}\text { Alter, } \\
\text { Geschlecht }\end{array}$} & \multicolumn{3}{|c|}{ Personen insgesamt } & \multicolumn{3}{|c|}{ Personen in 1-Personen-Haushalten } \\
\hline & $\begin{array}{l}\text { Verarmungs- } \\
\text { quote' }^{1}\end{array}$ & $\begin{array}{l}\text { relativer } \\
\text { Einkommens- } \\
\text { ausfall }{ }^{2}\end{array}$ & $\begin{array}{l}\text { absoluter } \\
\text { Wohlstands- } \\
\text { verlust }^{3}\end{array}$ & $\begin{array}{l}\text { Verarmungs- } \\
\text { quote }^{1}\end{array}$ & $\begin{array}{l}\text { relativer } \\
\text { Einkommens- } \\
\text { ausfa } \|^{2}\end{array}$ & $\begin{array}{l}\text { absoluter } \\
\text { Wohlstands- } \\
\text { verlust }^{3}\end{array}$ \\
\hline $\begin{array}{l}\text { Dim } \\
\text { Spalte }\end{array}$ & $\begin{array}{c}\% \\
1\end{array}$ & $\begin{array}{c}\% \\
2\end{array}$ & $\begin{array}{c}\text { Sh.-Vielf. } \\
3\end{array}$ & $\begin{array}{c}\% \\
4\end{array}$ & $\begin{array}{c}\% \\
5\end{array}$ & $\begin{array}{c}\text { Sh.-Vielf. } \\
6\end{array}$ \\
\hline Insgesamt & 9,9 & 20,4 & 0,42 & 10,8 & 29,4 & 0,71 \\
\hline $\begin{array}{r}-25 \\
26-30 \\
31-35\end{array}$ & $\begin{array}{r}13,2 \\
6,8 \\
5,8\end{array}$ & $\begin{array}{l}21,2 \\
21,0 \\
20,3\end{array}$ & $\begin{array}{l}0,39 \\
0,45 \\
0,44\end{array}$ & $\begin{array}{l}6,3 \\
8,3\end{array}$ & $\begin{array}{l}31,2 \\
30,9\end{array}$ & $\begin{array}{l}0,66 \\
0,77\end{array}$ \\
\hline $\begin{array}{l}36-40 \\
41-45\end{array}$ & $\begin{array}{r}9,1 \\
10,5\end{array}$ & $\begin{array}{l}20,4 \\
18,8\end{array}$ & $\begin{array}{l}0,44 \\
0,40\end{array}$ & 12,7 & 29,8 & 0,80 \\
\hline $\begin{array}{l}46-50 \\
51-55\end{array}$ & $\begin{array}{l}7,7 \\
9,0\end{array}$ & $\begin{array}{l}18,2 \\
20,9\end{array}$ & $\begin{array}{l}0,41 \\
0,42\end{array}$ & & & \\
\hline $56-60$ & 14,6 & 23,9 & 0,52 & 14,5 & 26,8 & 0,66 \\
\hline $61-65$ & 0,0 & 16,8 & 0,38 & & & \\
\hline $66 \mathrm{u} . \mathrm{m}$. & 8,5 & 13,4 & 0,21 & & & \\
\hline Männer & 11,7 & 22,7 & 0,47 & 7,3 & 32,1 & 0,81 \\
\hline-25 & 14,3 & 22,6 & 0,43 & 0,0 & 39,7 & 0,83 \\
\hline $\begin{array}{l}26-30 \\
31-35\end{array}$ & $\begin{array}{l}8,7 \\
7,5\end{array}$ & $\begin{array}{l}22,9 \\
22,6\end{array}$ & $\begin{array}{l}0,48 \\
0,49\end{array}$ & 10,0 & 32,0 & 0,81 \\
\hline $\begin{array}{l}36-40 \\
41-45\end{array}$ & $\begin{array}{l}12,9 \\
14,2\end{array}$ & $\begin{array}{l}24,0 \\
22,8\end{array}$ & $\begin{array}{l}0,52 \\
0,46\end{array}$ & 14,0 & 31,6 & 0,88 \\
\hline $\begin{array}{l}46-50 \\
51-55 \\
56-60 \\
61-65 \\
66 \mathrm{u} . \mathrm{m} .\end{array}$ & $\begin{array}{r}10,8 \\
12,4 \\
10,8 \\
0,0 \\
6,9\end{array}$ & $\begin{array}{l}20,9 \\
23,3 \\
23,8 \\
19,0 \\
16,4\end{array}$ & $\begin{array}{l}0,46 \\
0,48 \\
0,51 \\
0,39 \\
0,25\end{array}$ & 0,0 & 25,4 & 0,75 \\
\hline Frauen & 7,1 & 16,8 & 0,35 & 14,0 & 27,1 & 0,62 \\
\hline $\begin{array}{r}-25 \\
26-30\end{array}$ & $\begin{array}{r}12,0 \\
41\end{array}$ & $\begin{array}{l}19,6 \\
182\end{array}$ & $\begin{array}{l}0,35 \\
0,41\end{array}$ & 10,1 & 25,4 & 0,55 \\
\hline $\begin{array}{l}26-30 \\
31-35\end{array}$ & $\begin{array}{l}4,1 \\
2,5\end{array}$ & $\begin{array}{l}18,2 \\
15,2\end{array}$ & $\begin{array}{l}0,41 \\
0,35\end{array}$ & 7,2 & 29,5 & 0,70 \\
\hline $\begin{array}{l}36-40 \\
41-45\end{array}$ & $\begin{array}{l}2,8 \\
4,3\end{array}$ & $\begin{array}{l}14,4 \\
12,1\end{array}$ & $\begin{array}{l}0,30 \\
0,30\end{array}$ & 7,9 & 25,6 & 0,61 \\
\hline $\begin{array}{l}46-50 \\
51-55 \\
56-60 \\
61-65 \\
66 \text { u. m. }\end{array}$ & $\begin{array}{c}2,0 \\
2,8 \\
20,9 \\
/ \\
(11,3)\end{array}$ & $\begin{array}{c}13,1 \\
16,5 \\
24,1 \\
1 \\
(8,1)\end{array}$ & $\begin{array}{c}0,32 \\
0,31 \\
0,53 \\
/ \\
(0,14)\end{array}$ & $(20,0)$ & 27,3 & 0,63 \\
\hline
\end{tabular}

' Die Verarmungsquote gibt den Anteil der bei Arbeitslosigkeit Verarmenden an den bei Erwerbstätigkeit nicht armen Arbeitern und Angestellten wieder.

2 in $\%$

3 in Vielfachen der Sozialhilfeschwelle

Quelle: Transferumfrage 1981; eigene Berechnungen 
höheren Bildungsschicht nur kürzer andauern dürfte und generell eine maximale Einkommenskompensation durch Arbeitslosengeld, Wohngeld und Steuersplitting unterstellt wurde, obwohl obere Bildungsschichten $u$. U. eine geringere Dunkelziffer der Nichtinanspruchnahme aufweisen und durch stabilere Beschäftigung häufiger und länger arbeitslosengeldberechtigt sind. In dem seltener eintretenden und seltener anhaltenden Fall von Arbeitslosigkeit sind aber dennoch die oberen Bildungsschichten bei gleichem Zugang zu Arbeitslosengeld, Wohngeld und Steuersplitting in demselben Ausmaß wie die unteren Bildungsschichten verarmungsgefährdet.

\subsection{Arbeitslosigkeitsrisiko versus Verarmungsrisiko im Fall von Arbeitslosigkeit}

Heinze et al. (1981; 1983) postulieren schließlich, daß sich die Arbeitsmarktsegmentierung - zusätzlich zu dem segmentspezifischen Verarmungsrisiko bei Arbeitslosigkeit - auch durch segmentspezifisch unterschiedliche Arbeitslosigkeitsrisiken auf die Armutsgefährdung auswirkt. Im sekundären Segment findet somit eine Kumulation von Nachteilen statt: eine Verbindung von unsicheren Arbeitsplätzen und erhöhtem Verarmungsrisiko in dem Fall, daß Arbeitslosigkeit eintritt. Diese dritte Hypothese soll hier unter Bezug auf die vorher diskutierten Ergebnisse zum Verarmungsrisiko bei Arbeitslosigkeit (Punkt 4.2) und unter Bezug auf amtliche spezifische Arbeitslosenquoten beleuchtet werden. Die Transferumfrage erlaubt selbst keine repräsentative Wiedergabe von Arbeitslosenquoten. Leider setzen aber auch die amtlich ausgewiesenen Arbeitslosenquoten vergleichsweise enge Grenzen. Spezifische Arbeitslosenquoten werden in aller Regel nur eindimensional, aufgeschlüsselt nach einem einzigen Merkmal, ausgewiesen. Eine dreidimensionale Aufgliederung nach Ausbildung, Alter und Geschlecht existiert gar überhaupt nicht. Die zum Zweck der nachfolgenden Untersuchung vergleichsweise beste Berücksichtigung gruppenspezifischer Arbeitslosenquoten läßt sich für das Untersuchungsjahr der Transferumfrage aus Berechnungen von Tessaring (1982) ableiten, die sich immerhin gleichzeitig auf Geschlechts- und Ausbildungsgruppen beziehen. Die Arbeitsmarktsegmentierung wird deshalb in diesem Abschnitt nur anhand von Geschlecht und Ausbildung operationalisiert.

Die nach Geschlecht und Ausbildung unterschiedliche Arbeitslosigkeitsbetroffenheit ist im oberen Teil von Tabelle 7 wiedergegeben. Die Spalten 1 bis 3 enthalten die (damals noch vergleichsweise niedrigen) Arbeitslosenquoten des Jahres $1981 .^{9}$ Die Arbeitslosenquote betrug im September 1981 durchschnittlich 4,4\%. Deutlich höher lag sie bei denjenigen ohne Berufsausbildung. Ebenfalls sind Frauen in allen Ausbildungsgruppen stärker von Arbeitslosigkeit betroffen als Männer. Die Geschlechts- und Ausbildungsdifferenzierung der Arbeitslosenquoten ist noch deutlicher aus dem rechten oberen Teil von Tabelle 7 (Spalten 4 bis 6) zu erkennen. Während Männer nur $80 \%$ der durchschnittlichen Arbeitslosenquote aufweisen, sind Frauen mit $132 \%$ stark überdurchschnittlich von Arbeitslosigkeit betroffen. Frauen ohne Berufsausbildung sind gar mit $195 \%$ fast der doppelten Arbeitslosenquote ausgesetzt. Man kann wohl davon ausgehen, daß diese Strukturen auch auf dem inzwischen weit höheren Arbeitslosigkeitsniveau des Jahres 1987 weitgehend erhalten geblieben sind. Im wesentlichen kann man daher, was die Verteilung der Arbeitslosigkeit anbetrifft, von einer Bestätigung der arbeitsmarktsegmenttheoretischen Vorstellungen ausgehen. Erstaunlich ist allerdings, daß die Arbeitslosigkeit unter den Hochund den Fachhochschulabsolventen durchaus nicht am geringsten ist; hier schlägt bereits die Jungakademikerarbeitslosigkeit zu Buche.

Im mittleren Abschnitt von Tabelle 7 sind die zum Teil schon aus Tabelle 6 bekannten Verarmungsquoten im Fall von Arbeitslosigkeit nach Geschlecht und Ausbildungsabschluß aufgeschlüsselt, wobei diejenigen, die keinem Ausbildungsabschluß zugeordnet werden konnten (anders als in Tabelle 6), auch im Gesamtdurchschnitt unberücksichtigt blieben. Die Angaben gehen (wie schon in Punkt 4.2) von dem hypothetischen Fall von Arbeitslosigkeit aus. Eine systematische Ausbil-

${ }^{9}$ Die Angaben beziehen sich auf September 1981. Zusätzlich ist $z u$ berücksichtigen, daß sich die amtlicher seits veröffentlichten Arbeitslosenquoten immer auf alle abhängig Beschäftigten plus Erwerbslosen, also auch alle Beamten, beziehen. Umgerechnet auf die Arbeiter- und Angestelltenschaft, die überhaupt nur von Arbeitslosigkeit betroffen werden kann, betrug die Arbeitslosenquote im Gesamtdurchschnitt auch 1981 (September) schon 5\%, wenn man einen Beamtenanteil unter den abhängig Beschäftigten von $10 \%$ unterstellt (Statistisches Bundesamt. Fachserie 1, Reihe 4.1.1 (1982): 60; eigene Berechnungen). Da die in den einzelnen Geschlechts-Ausbildungs-Kombinationen wahrscheinlich unterschiedlichen Beamtenquoten unbekannt sind und Strukturverzerrungen deshalb ohnehin nicht bereinigt werden konnten, wurde auf Um rechnungen der genannten Art generell verzichtet. 
dungsdifferenzierung der Verarmungsquoten bei Arbeitslosigkeit ist, wie schon gesagt, nicht festzustellen. Hinsichtlich der Geschlechtsdifferenzierung erscheinen Frauen, wenn man von Arbeitslosıgkeit ausgeht, einem deutlich geringeren Verarmungsrisiko ausgesetzt als Männer. Während also die Verteilung des Arbeitslosigkeitsrisikos weitgehend den Hypothesen der Segmentierungstheorie entspricht, ist hinsichtlich des Verarmungsrisikos im Fall schon existierender Arbeitslosigkeit aufgrund unterschiedlicher Familienzusammenhänge eher von einem umgekehrten Zusammenhang aus- zugehen. Es stellt sich daher die Frage, ob letztlich die der Arbeitsmarktsegmentierung entsprechende Verteilung des Arbeitslosigkeitsrisikos oder die doch stärker durch den Familienkontext geprägte Verteilung des Verarmungsrisikos bei Arbeitslosigkeit auf die Verarmung durch Arbeitslosigkeit einwirken.

Die Kumulation von Arbeitslosigkeits- und Verarmungsrisiko ist aus dem unteren Teil von Tabelle 7 zu erkennen. Die dort wiedergegebenen Werte umfassen sowohl das Arbeitslosigkeitsrisiko wie

Tabelle 7 Arbeitslosigkeit und Verarmung nach Berufsausbildung und Geschlecht.

\begin{tabular}{|c|c|c|c|c|c|c|}
\hline \multirow[t]{2}{*}{ Ausbildungsabschluß } & \multicolumn{3}{|c|}{$\begin{array}{l}\text { Arbeitslosigkeitsquoten/Verarmungs- } \\
\text { quoten }{ }^{1}\end{array}$} & \multicolumn{3}{|c|}{ Relative Betroffenheit ${ }^{2}$} \\
\hline & Insgesamt & Männer & Frauen & Insgesamt & Männer & Frauen \\
\hline \multirow[t]{2}{*}{ Spalte } & 1 & 2 & 3 & 4 & 5 & 6 \\
\hline & \multicolumn{3}{|c|}{ Arbeitslosigkeitsrisiko } & & & \\
\hline Insgesamt & 4,4 & 3,5 & 5,8 & 1 & 0,80 & 1,32 \\
\hline ohne Ausbildung & 8,3 & 8,0 & 8,6 & 1,89 & 1,82 & 1,95 \\
\hline Lehre & 3,0 & 2,4 & 4,0 & 0,68 & 0,55 & 0,91 \\
\hline Berufsfachschule & 3,0 & 2,4 & 4,0 & 0,68 & 0,55 & 0,91 \\
\hline \multicolumn{7}{|l|}{ Fach-, Meister-, Techniker- } \\
\hline Fachhochschule & 2,4 & 1,9 & 4,3 & 0,55 & 0,43 & 0,98 \\
\hline Universität & 2,4 & 1,9 & 3,4 & 0,55 & 0,43 & 0,77 \\
\hline \multicolumn{7}{|c|}{ Verarmungsrisiko im Fal/ von Arbeitslosigkeit } \\
\hline Insgesamt ${ }^{3}$ & 7,7 & 9,1 & 5,5 & 1 & 1,26 & 0,58 \\
\hline ohne Ausbildung & 7,4 & 10,8 & 5,0 & 0,96 & 1,40 & 0,65 \\
\hline Lehre & 8,2 & 10,2 & 4,3 & 1,06 & 1,32 & 0,56 \\
\hline Berufsfachschule & 4,3 & 3,7 & 4,8 & 0,56 & 0,48 & 0,62 \\
\hline $\begin{array}{l}\text { Fach-, Meister-, Techniker- } \\
\text { schule }\end{array}$ & 7,8 & $(8,1)$ & $(5,9)$ & 1,01 & $(1,05)$ & $(0.77)$ \\
\hline Fachhochschule & 3,6 & 2,8 & $(7,2)$ & 0,47 & 0,36 & $(0,94)$ \\
\hline Universität & 8,4 & $(11,9)$ & $(0,0)$ & 1,09 & $(1,55)$ & $(0,00)$ \\
\hline \multicolumn{7}{|c|}{ Risiko der Verarmung durch eintretende Arbeitslosigkeit } \\
\hline Insgesamt $^{3}$ & 0,34 & 0,35 & 0,32 & 1 & 1,03 & 0,94 \\
\hline ohne Ausbildung & 0,61 & 0,86 & 0,43 & 1,80 & 2,54 & 1,27 \\
\hline Lehre & 0,25 & 0,24 & 0,17 & 0,74 & 0,71 & 0,50 \\
\hline $\begin{array}{l}\text { Berufsfachschule } \\
\text { Fach-, Meister-, Techniker- }\end{array}$ & 0,13 & 0,09 & 0,19 & 0,38 & 0,27 & 0,56 \\
\hline schule & 0,14 & $(0,06)$ & $(0,36)$ & 0,41 & $(0,18)$ & $(1,06)$ \\
\hline Fachhochschule & 0,09 & 0,05 & $(0,31)$ & 0,27 & 0,15 & $(0,92)$ \\
\hline Universität & 0,20 & $(0,23)$ & $(0,00)$ & 0,59 & $(0,68)$ & $(0,00)$ \\
\hline
\end{tabular}

in $\%$

in Vielfachen der durchschnittlichen Arbeitslosen- bzw. Verarmungsquole

ohne diejenigen mit anderen Abschlüssen, in Ausbildung und ohne Angabe (s. a. Tabelle 3)

Ergebnisse, die auf Fallzahlen unter 25 beruhen, sind in Klammer wiedergegeben.

Quelle: Tessaring 1982: 25f; Transferumfrage 1981; eigene Berechnungen 
auch das der Verarmung im Fall von Arbeitslosigkeit. Das gesamtdurchschnittliche Risiko der Verarmung durch Arbeitslosigkeit betrug beispielsweise im Jahre 1981 0,34\%, wobei sich das der Arbeitslosigkeit auf $4,4 \%$ und das der Verarmung im Fall von Arbeitslosigkeit auf $7,7 \%$ beläuft $(4,4 \%$ $\times 7,7 \%=0,34 \%)$. Dieser Wert dürfte sich bis heute, bei einem mehr als doppelten Arbeitslosigkeitsrisiko, ebenfalls mehr als verdoppelt haben, wenn man von einem gleich gebliebenen Verarmungsrisiko im Fall von Arbeitslosigkeit ausgeht. Außerdem ist zu berücksichtigen, daß es sich aufgrund der unterstellten maximalen Einkommenskompensation durch Arbeitslosengeld, Wohngeld und Steuersplitting um einen Mindestwert handelt, der insbesondere für die erste Zeit der Arbeitslosigkeit von Bedeutung ist. Die seit 1981 beobachtete Zunahme der Sozialhilfefälle beruht auch auf einer länger gewordenen Dauer der Arbeitslosigkeit und einer Zunahme des Verarmungsrisikos bei Arbeitslosigkeit. Männer sind wie gesagt einem geringeren Arbeitslosigkeitsrisiko, in dem dennoch eintretenden Fall von Arbeitslosigkeit aber einem höheren Risiko der Verarmung ausgesetzt als Frauen. Wie der Kumulation beider Risiken zu entnehmen, sind Männer und Frauen mit 0,35 bzw. $0,32 \%$ summa summarum etwa demselben Risiko der Verarmung durch Arbeitslosigkeit ausgesetzt. Man muß hier eher von einer kompensierenden denn von einer kumulierenden Risikostruktur sprechen. Hinsichtlich des Geschlechts nivellieren sich offenbar Arbeitsmarkt- und familiale Einflüsse. Was andererseits die Differenzierung nach dem beruflichen Ausbildungsabschluß anbelangt, gibt - von den Hochschulabsolventen abgesehen $^{10}-$ doch der Arbeitsmarkt den Ausschlag.

\section{Zusammenfassung und Ausblick}

In dem vorliegenden Beitrag wurde der Frage nachgegangen, inwieweit aus der Stellung auf dem Arbeitsmarkt auch auf die Gefahr von Armut geschlossen werden kann, die früher hauptsächlich unter dem Einfluß familialer Faktoren analysiert wurde. Im wesentlichen wurden erstmals auf der Basis einer empirischen Mikrosimulation drei Fra-

10 Das bei Hochschulabsolventen vergleichsweise hohe kumulierte Risiko der Verarmung durch Arbeitslosigkeit sollte nicht überinterpretiert werden. U.U. spielt hier auch das Alter eine entscheidende Rolle, dessen Einfluß jedoch nicht untersucht werden konnte. gen untersucht. Welchen Einfluß haben Arbeitsmarktsegmentierung versus Familie auf die sog. Erwerbstätigenarmut? Wie wirken sich beide Faktoren auf das Risiko von Verarmung und Abstieg im Fall von Arbeitslosigkeit aus? Inwieweit kumulieren Arbeitslosigkeits- und Verarmungsrisiken im Hinblick auf Merkmale der Arbeitsmarktstellung und des Familienzusammenhangs?

Im Ergebnis läßt sich festhalten, daß die Erwerbstätigenarmut und das Verarmungsrisiko bei Arbeitslosigkeit - trotz deutlicher Segmentierungseinflüsse - stark auch von dem familialen Kontext bestimmt werden. Hinsichtlich der Verknüpfung von Arbeitslosigkeits- und Verarmungsrisiko im Fall von Arbeitslosigkeit kann man sogar teilweise von kompensierenden Einflüssen ausgehen.

\section{Literatur}

Adamy, W./Steffen, J., 1984: Arbeitslos gleich arm Ursachen und Lösungsansätze zur Beseitigung der Neuen Armut. In: WSI-Mitteilungen, S. 574-581

Albrecht, R./Hautsch, G./Reidegeld, E., 1977: Arbeitslosigkeit als Zumutung. Bemerkungen zu Umfang und Folgen der Arbeitslosigkeit. In: Sozialer Fortschritt, S. 281-285

Bäcker, G., 1983: Arbeitsmarktlage, Einkommensentwicklung und Verarmung. In: Blätter der Wohlfahrtspflege, S. 266-268

Balsen, W./Nakielski, H./Rössel, K./Winkel, R., 1984: Die neue Armut: Ausgrenzung von Arbeitslosen aus der Arbeitslosenunterstützung. Bund, Köln

Breuer, W./Schoor-Theissen, J./Silbereisen, R. K., 1984: Auswirkungen der Arbeitslosigkeit auf die Situation der betroffenen Familien. Statistikauswertung und Literaturstudie. Erstellt durch das Institut für Sozialforschung und Gesellschaftspolitik e. V. im Auftrag des Bundesministers für Jugend, Familie und Gesundheit. Köln

Brinkmann, Ch., 1976: Finanzielle und psycho-soziale Belastungen während der Arbeitslosigkeit. In: Mitteilungen aus der Arbeitsmarkt- und Berufsforschung, S. $397-413$

Brinkmann, Ch., 1984: Die individuellen Folgen langfristiger Arbeitslosigkeit. Ergebnisse einer repräsentativen Längsschnittuntersuchung. In: Mitteilungen aus der Arbeitsmarkt- und Berufsforschung. S. 454-473

Brinkmann, Ch./Schober, K., 1982: Methoden und erste Ergebnisse aus der Verlaufsuntersuchung des IAB bei Arbeitslosen (Zugänge November 1981). In: Mitteilungen aus der Arbeitsmarkt- und Berufsforschung, S. 408-425

Brinkmann, Ch./Spitznagel, E., 1984: Belastungen des Haushalts durch Arbeitslosigkeit. In: Hauswirtschaft und Wissenschaft, S. 256-263 
Büchtemann, C. F., 1982: Erwerbskarrieren im Anschluß an Arbeitslosigkeit. In: Mitteilungen aus der Arbeitsmarkt- und Berufsforschung, S. 120-130

Büchtemann, C. F., 1985: Soziale Sicherung bei Arbeitslosigkeit und Sozialhilfebedürftigkeit. Datenlage und neue Befunde. In: Mitteilungen aus der Arbeitsmarktund Berufsforschung, S. 450-466

Büchtemann, C. F./von Rosenbladt, B., 1981: Arbeitslose 1978. Die Situation in der Arbeitslosigkeit. In: Mitteilungen aus der Arbeitsmarkt- und Berufsforschung, S. 22-38

Büchtemann, C. F./von Rosenbladt, B., 1983: Kumulative Arbeitslosigkeit. In: Mitteilungen aus der Arbeitsmarkt- und Berufsforschung, S. 262-275

Buttler, F./Gerlach, K./Liepmann, P., 1978: Über den Zusammenhang von Arbeitsmarkt und Armut - Das Alte an der "Neuen sozialen Frage“. In: H. P. Widmaier (Hrsg.), Zur neuen sozialen Frage. Duncker u. Humblot, Berlin, S. 9-31

Cramer, U., 1980: Anpassungsvorgänge bei der Besetzung von offenen Stellen durch Arbeitslose. In: Mitteilungen aus der Arbeitsmarkt- und Berufsforschung, S. $116-124$

Cremer-Schäfer, H., 1981: Arbeitslosigkeit, Einkommensverlust und Armut. Sfb 3-Arbeitspapier Nr. 54, Frankfurt, Mannheim

Doeringer, P. B./Piore, J. M., 1971: International Labor Markets and Manpower Analysis. C. D. Health, Lexington, Massachusetts

Furmaniak, K., 1984: West Germany: Poverty, Unemployment and Social Insurance. In: R. Walker/R. Lawson/P. Townsend (Hrsg.), Responses to Poverty. Lessons from Europe. Heinemann, London, S. 136-159

Geißler, H., 1976: Die neue soziale Frage. Herder, Freiburg

Gölter, G., o. J.: Begleiter der Arbeitslosigkeit: Abstieg und Armut. Dokumentation zur wirtschaftlichen Lage von Arbeitslosen in der Bundesrepublik Deutschland. Ministerium für Soziales, Gesundheit und Sport, Rheinland-Pfalz

Hauser, R./Fischer, I./Klein, Th., 1985: Verarmung durch Arbeitslosigkeit? In: St. Leibfried/F. Tennstedt (Hrsg.), Politik der Armut und die Spaltung des Sozialstaats. Suhrkamp, Frankfurt, S. 213-248; eine ausführlichere Fassung ist als Sfb 3-Arbeitspapier Nr. 154 erschienen.

Heinze, R. G./Hohn, H.-W./Hinrichs, K./Olk, Th., 1981: Armut und Arbeitsmarkt. Zum Zusammenhang von Klassenlagen und Verarmungsrisiko im Sozialstaat. In: Zeitschrift für Soziologie, S. 219-243
Heinze, R. G./Hohn, H.-W./Hinrichs, K./Olk, Th., 1983: Armut - nur ein Restproblem des Sozialstaates? In: Zeitschrift für Soziologie, S. 157-168

Klein, Th., 1985: Umfang und Strukturen der Armut unter dem Einfluß von Zweitverdienern. In: R. Hauser/B. Engel (Hrsg.), Soziale Sicherung und Einkommensverteilung. Empirische Analysen für die Bundesrepublik Deutschland. Campus, Frankfurt, New York, S. $355-379$

Klein, Th., 1986: Äquivalenzskalen - ein Literatursurvey. Sfb 3-Arbeitspapier Nr. 195, Frankfurt, Mannheim

Klein, Th., 1987: Verarmung und sozialer Abstieg von Familien durch Arbeitslosigkeit. Eine mikroanalytische Untersuchung für die Bundesrepublik Deutschland. Campus, Frankfurt, New York

Kohl, J./Leisering, L., 1982: Armut und Arbeitsmarkt: Wo ist der Zusammenhang? In: Zeitschrift für Soziologie, S. 410-428

Kortmann, K., 1977: Ausmaß und Ursachen der Armut und Möglichkeiten ihrer Bekämpfung durch eine Arbeitsplatzgarantie. SPES-Arbeitspapier Nr. 75, Frankfurt

Muhr, G., 1984: Neue Armut - Ursachen und Auswege. Zugang zur Arbeitslosenversicherung erleichtern. In: Soziale Sicherheit, S. 225-228

Naegele, G., 1979: Verarmung durch Arbeitslosigkeit. In: WSI-Mitteilungen, S. 650-658

Pfriem, H., 1979: Konkurrierende Arbeitsmarkttheorien. Neoklassische, Duale und Radikale Ansätze. Campus, Frankfurt, New York

Rosenbladt, B. von/Büchtemann, C. F., 1980: Arbeitslosigkeit und berufliche Wiedereingliederung. In: Mitteilungen aus der Arbeitsmarkt- und Berufsforschung, S. $552-572$

Schäfer, D., 1983: Anpassung des Systems der sozialen Sicherung an Rezession und Unterbeschäftigung. In: Sozialer Fortschritt, S. 121-134

Scholz, W., 1983: Sozialhilfe und Arbeitslosigkeit. In: Nachrichtendienst des Deutschen Vereins für öffentliche und private Fürsorge, S. 423-429

Sengenberger, W. (Hrsg.), 1978: Der gespaltene Arbeitsmarkt. Probleme der Arbeitsmarktsegmentation. Campus, Frankfurt, New York

Wacker, A., 1977: Arbeitslosigkeit. Soziale und psychische Voraussetzungen und Folgen. 2. Aufl., Europäische Verlagsanstalt, Frankfurt, Köln

Welzmüller, R., 1982: Einkommensgefährdungen durch Arbeitslosigkeit. Zur finanziellen Lage der Arbeitslosen. In: WSI-Mitteilungen, S. 447-457 